\title{
Enterobacteria Producing Extended Spectrum Beta- Lactamases : 74 Cases in a General Hospital in France
}

\author{
Margaux Geier, MD \\ Department of Oncology, University Hospital of Brest, France \\ *Corresponding author: Margaux Geier, MD, Department of Oncology, University Hospital of Brest, France; Email: bgeier.cabtraverse@orange.fr
}

Received: March 22, 2019; Accepted: April 02, 2019; Published: April 25, 2019;

\begin{abstract}
Antibiotics used to treat zoonoses, such as campylobacteriosis and salmonellosis become less effective, new data from the European center for Disease Prevention and Control (ECDC) confirm this observation. Enterobacteria are the most common causes of community or nosocomial infections. Some of them produce beta-lactamases extended spectrum (ESBL) frequently associated with resistance to antibiotics. We conduct a retrospective study from positive levies of enterobacteria ESBL identified in adult patients hospitalized at the Quimper hospital, during the year 2016. 74 patients (48women) median age 80 years were included. $30 \%$ lifed in an institution, $21 \%$ had an history of infection of EBLSE, $62 \%$ had urinary tract infection, $12 \%$ intraabdominal infection, 9\% respiratory infections. Distribution of Enterobacteriaceae was: E. coli (73\%), E.cloacae complex (16\%), K.pneumoniae (5.4\%), Citrobacter freundii $(2.7 \%)$, Citrobacter koseri $(1.4 \%)$, Salmonella enterica $(1.4 \%)$. Sensitivity profiles of antibiotics strains are presented. The strategies of antibiotic therapy are detailed and discussed. The most used molecule was ceftriaxone for probalistic treatment then fluoroquinolone, carbapenems and amoxicillin- clavulanate for second line. The evolution was favorable for 64 patients, 8 deaths, 6 serious complications were notified.
\end{abstract}

Keywords: Enterobacteria beta-lactamases extended spectrum, ESBL, nosocomial infection, antibiotic resistance

\section{Introduction}

Enterobacteria are the most common causes of community or nosocomial infections. They are usually treated with beta - lactams, such as penicillins, broad-spectrum cephalosporins and carbapenems, or fluoroquinolones. For several decades, there has been a significant increase in the resistance of Enterobacteriaceae to these antibiotics, in particular Escherichia coli (E. Coli) and Klebsiella pneumoniae (K. pneumoniae), It is related to their large-scale use appropriately or excessively in humans but also in animals $[1,2]$.

The $\beta$ - lactamases extended spectrum (ESBL) are a large heterogeneous family of bacterial enzymes, mainly found in the Enterobacteriaceae family (secretory Enterobacteriaceae ESBL (EBLSE)). They are induced either by the acquisition of genetic material in the form of a plasmid most frequently, or by a chromosomal mutation. Both mechanisms give the affected bacteria the ability to hydrolyze a wide variety of beta - lactams. These enzymes do not hydrolyse cephamycins nor carbapenems, and are inhibited, to varying degrees, by $\beta$ - lactamase inhibitors (Annex 1). The presence of ESBL is frequently associated with resistance to fluoroquinolones and carbapenems often remain the first choice molecules to treat these infections with the risk of emerging resistances to this class and the spread of carbapenemase- producing enterobacteria (CPL) [3].

EBLSE can cause hospital and community infections [4] with the consequent high risk of clinical failure in probabilistic treatments with cephalosporins or quinolones. The eur broadcast is now a major concern of health institutions in the fight against multi-resistant bacteria (BMR).

\section{Epidemiological data}

Discovered in the 80s in Europe, the first ESBL were of TEM

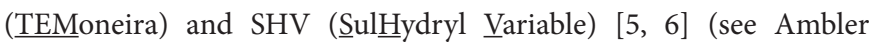
Classification, Annex 2). The majority were K. pneumoniae and were mainly responsible for nosocomial infections. Since now for several years, there is a worldwide spread of ESBL, in particular

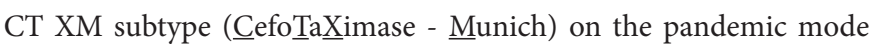
with a predominance of $E$. coli as a host bacterium, which is largely implicated in urinary tract infections $[7,8]$. In France, the incidence of EBLSE is increasing and has overtaken that of girls Staphylococcus aureus resistant to methicillin (MRSA) in hospitals (Figures 1 and 2; Annex 3 and 4).

Europe is characterized by a north-south and west-east distribution gradient [10,11] (Figures 3 and 4).

\section{Objectives of the study}

We conducted a retrospective study from positive levies of enterobacteriaceae producer extended-spectrum $\beta$-lactamase, identified in adult patients hospitalized at the Quimper hospital, during the year 2016. The main objective of this study was to evaluate in current practice the therapies administered and their efficacy in case of infection documented at EBLSE, and in particular to study alternative molecules to carbapenems. The secondary objectives were to identify the risk factors presented by these patients and to study the management of the infectious episode. 


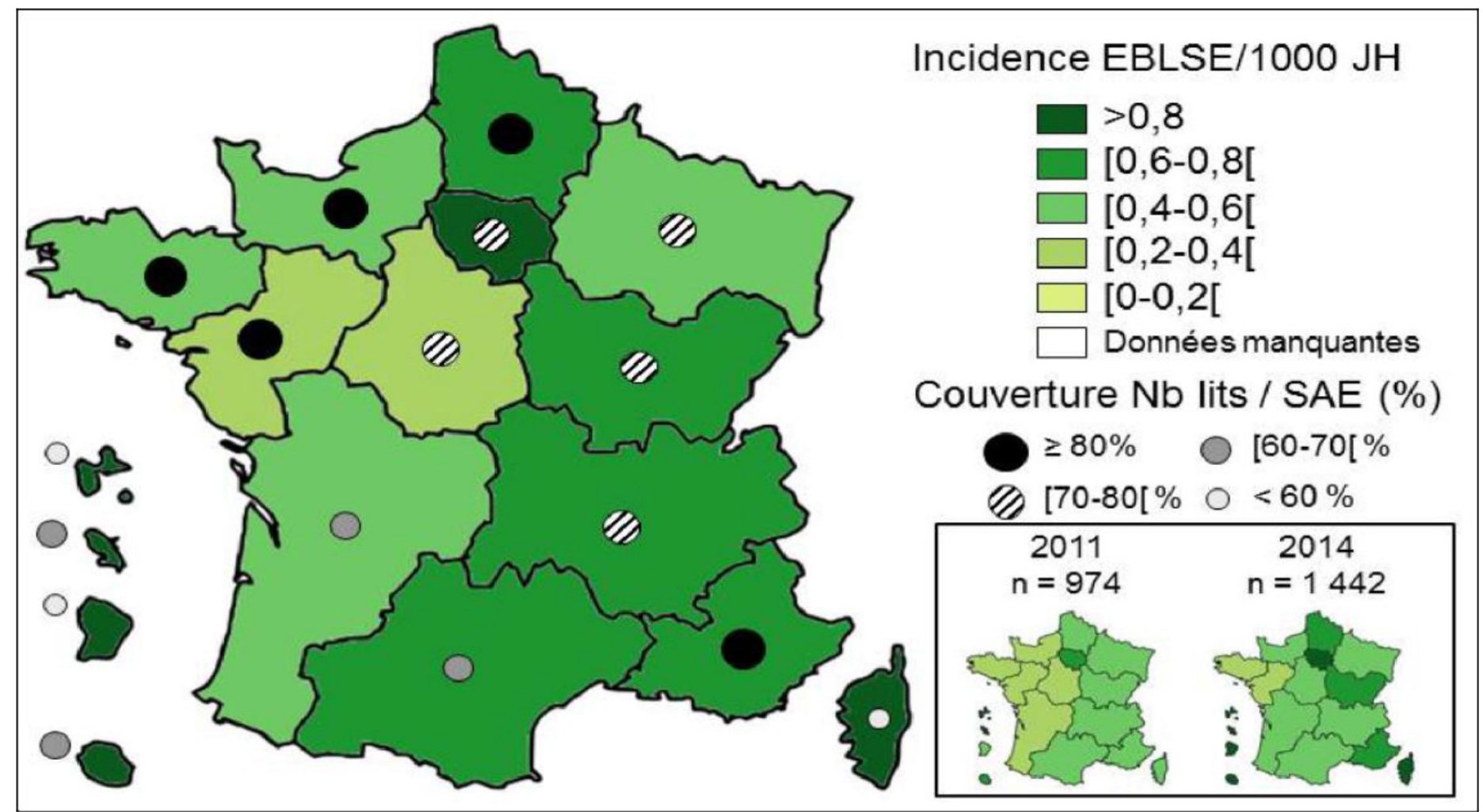

Figure 1. BMR-Raisin 2015 - Overall EBLSE Impacts per 1, 000 Hospital Days (JH) (all Health Facilities, n= 1, 427) by region.

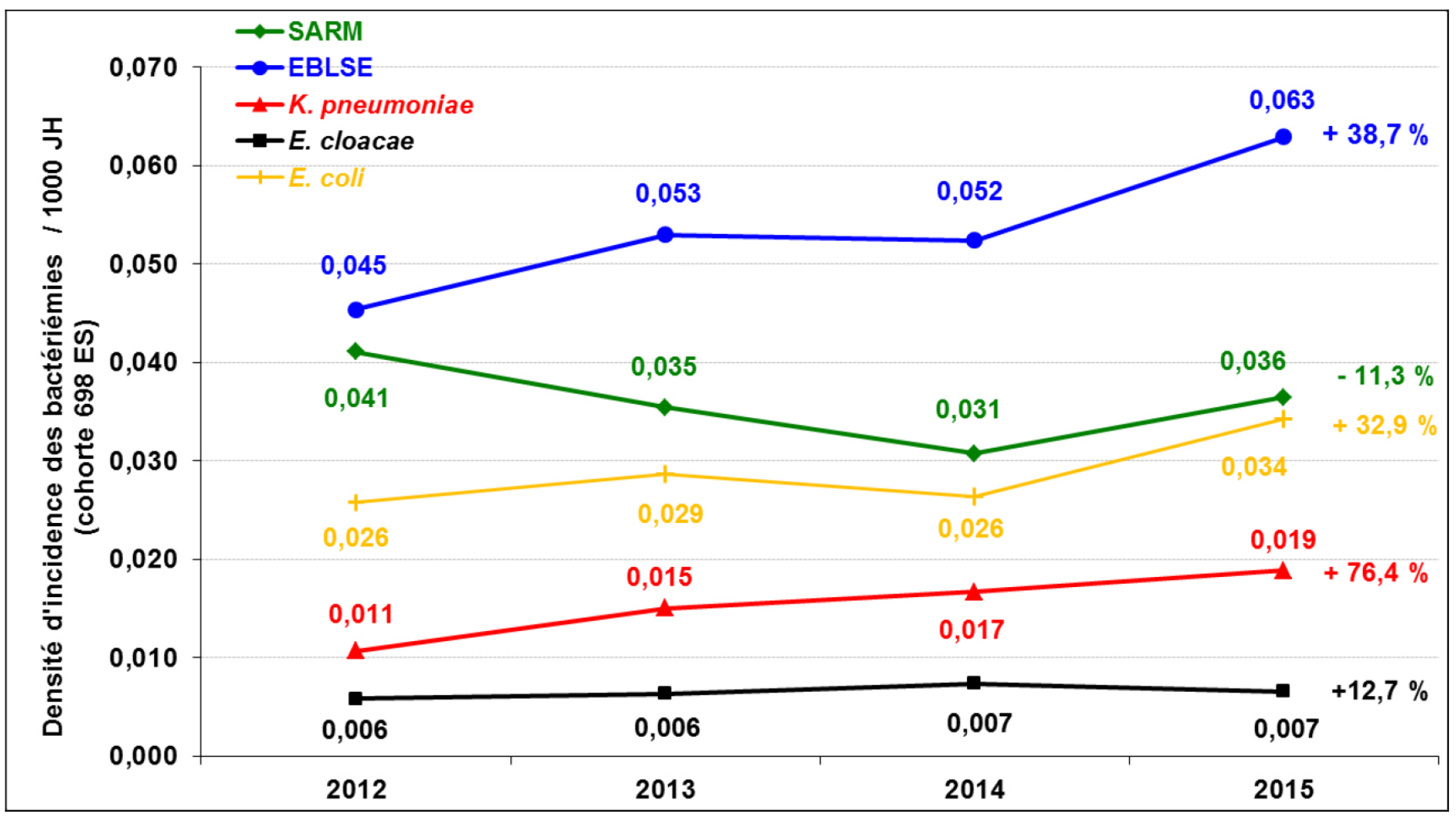

Figure 2. BMR-Raisin 2015 - Densities of MRSA and different EBLSE bacteremia incidence per 1, $000 \mathrm{JH}$ (annual overall incidence density born) between 2012 and 2015 (n = 698) 


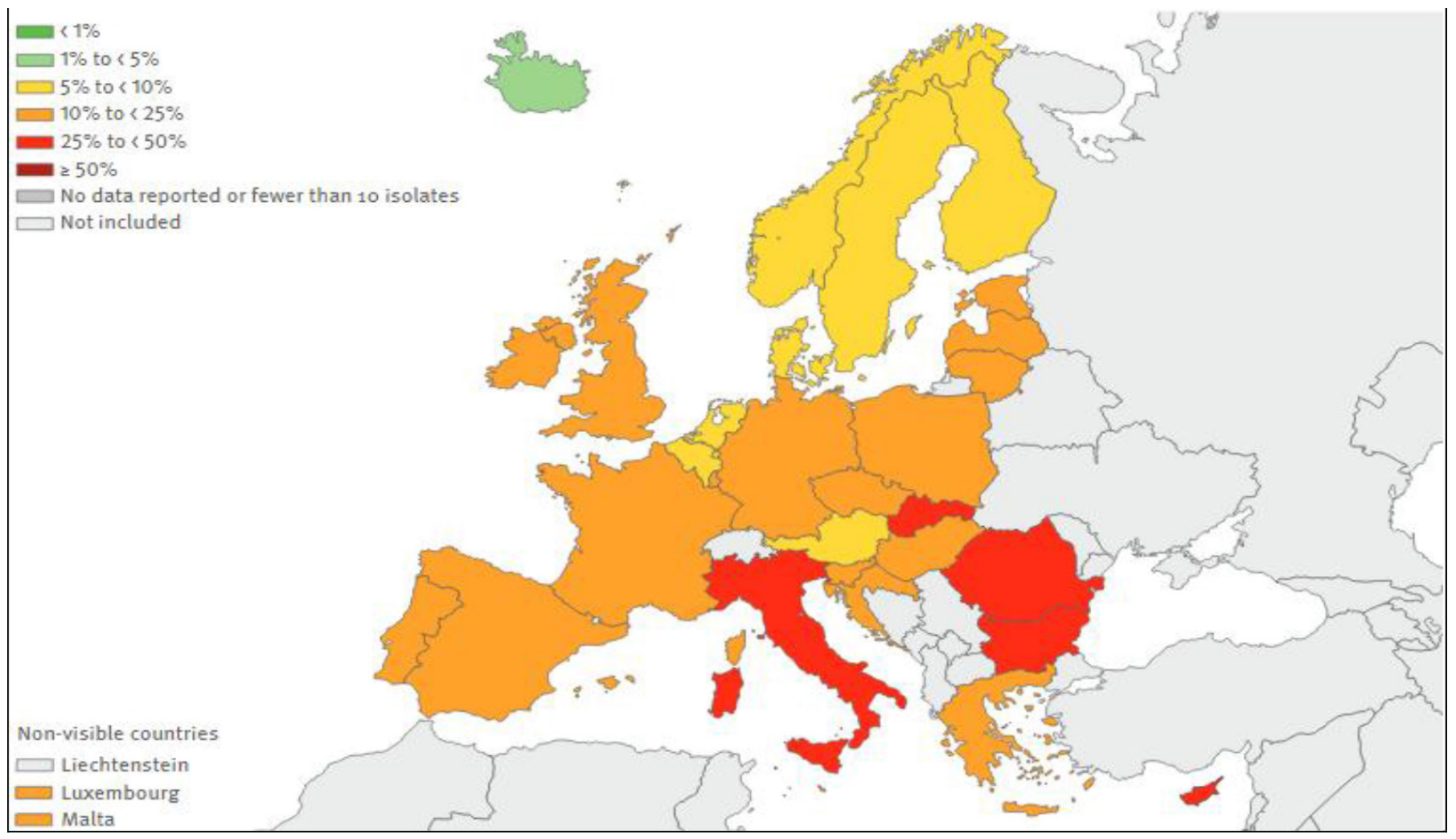

Figure 3. E. Coli. Percentage (\%) of invasive isolates with resistance to third- generation cephalosporins, by country, EU/EEA countries, European Centre for Disease Prevention and Control, Antimicrobial resistance surveillance in Europe, 2015

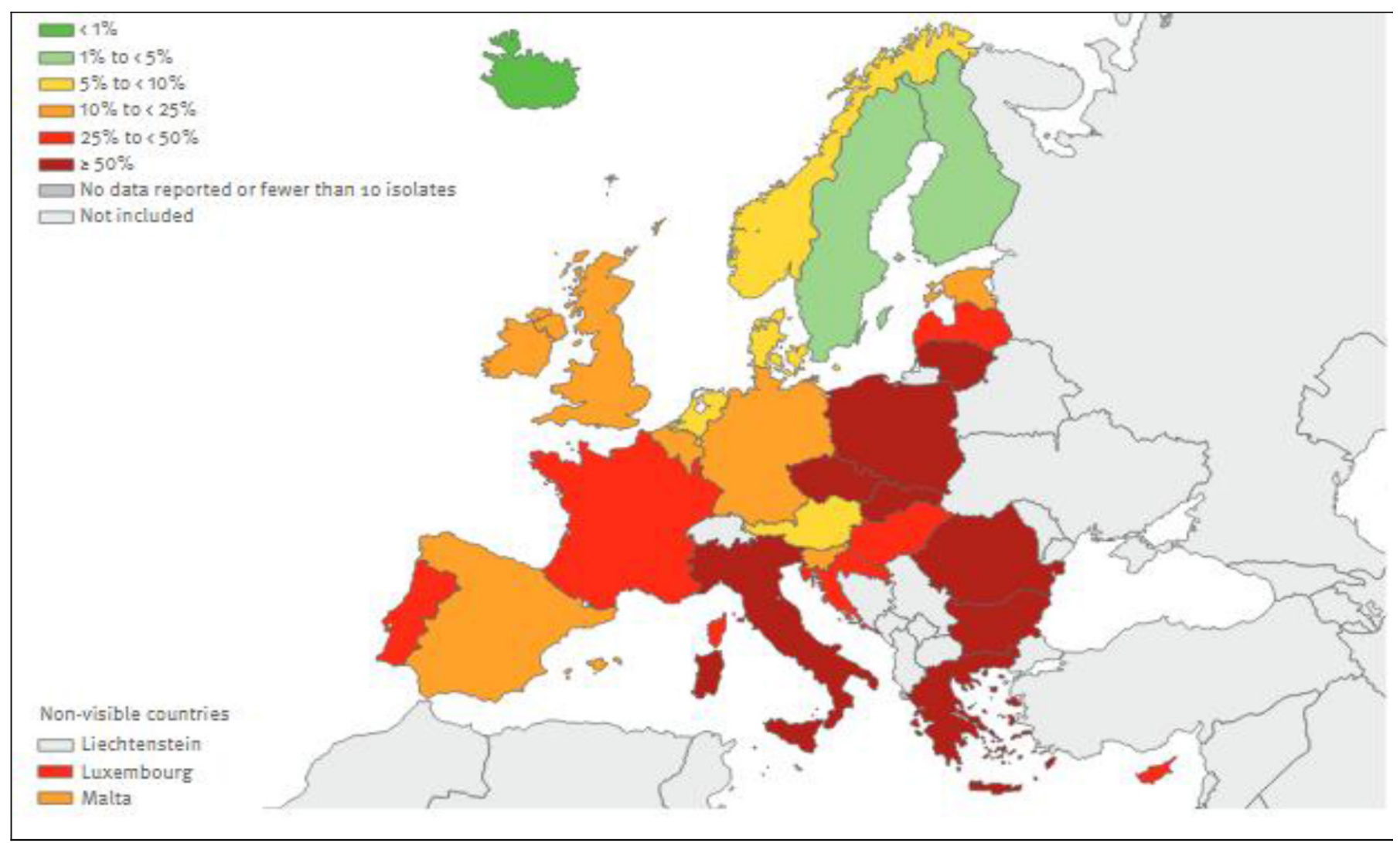

Figure 4. K. pneumoniae. Percentage (\%) of invasive isolates with resistance to third-generation cephalosporins, by country, EU/EEA countries, European Centre for Disease Prevention and Control, Antimicrobial resistance surveillance in Europe, 2015 


\section{Method}

This is a retrospective observational monocentric study, conducted at the Hospital Center of Quimper, France (population 100000 inhabitants)

\section{Study population and definitions}

The study population is for adults over 15 years old hospitalized during 2016.

\section{- Inclusion criteria:}

Included are EBLSE strains isolated from diagnostic specimens made during the survey period in hospitalized patients complete "(Short or long stay), but also outpatients consulting at the hospital or hospitalized for a total period of less than 24 hours. For the definition of EBLSE, the reference is the annual bulletin of the Committee of the antibiogram of the French Society of Microbiology (CA-SFM).

\section{- Exclusion criteria:}

Have been excluded (a) strains of EBLSE isolated from ecological samples (eg nose, stool...), that is to say in which we exclusively look for multiresistant bacteria (for example by using selective media containing antibiotics), (b) duplicates defined as strains isolated in a patient for which a strain of the same species and the same antibiotype (same antibiotype $=$ no major difference in terms of clinical categories $[\mathrm{S}>\mathrm{R}$ or $\mathrm{R}>\mathrm{S}$ ] for antibiotics on the standard list defined by the CASFM) has already been taken into account during the survey period, regardless of the diagnostic sampling from which it was isolated.

Infection was associated with care if it occurred during or after management (diagnostic, therapeutic, palliative, preventive or educational) and was not present or incubated at the beginning of the medical care [12]. This definition was extended to patients residing in long-term care facilities, such as nursing homes for the elderly (EHPAD).

\section{ESBL risk factors}

Certain risk factors recognized as promoting the risk of developing EBLSE infection were sought in all patients. Among these factors, were retained :

- Older age

- Institutionalization

- A history of infection and / or colonization at EBLSE

- Long-term hospitalization

- Hospitalization in the year preceding admission

- A trip abroad during the past year

- Medical device in place

\section{The infectious episode}

Were notified: the type of infection and the presence of bacteremia; the different types of samples and EBLSE strains isolated for diagnostic purposes, characterized by family, genus, species and subspecies by the microbiologist; the context of care or community; complicated forms (septic shock, abscess...)

\section{Therapeutic}

The first antibiotic treatment administered was retained, with for each patient the type of drug or combination of drugs used and the duration of treatment. Antibiotic therapy was then said to be probabilistic when it was initiated before the nature and / or sensitivity of the microorganism (s) responsible for the infection were known. In case of modification of the therapeutics after reception of the antibiogram, this one was notified. Antibiotic therapy was then said to be documented when it was initiated after knowledge of the nature and / or sensitivity of the microorganism (s) responsible for the infection.

The use of carbapenems in probabilistic and a possible association with an aminoglycoside and the notion of an opinion taken with an infectious diseases specialist of the hospital center (HC) were also noted.

\section{Become patients}

The evolution was noted as favorable if the patient was considered cured at one month of the end of the treatment. An infectious complication was sought before, during or after antibiotic therapy. In the event of death, it was notified.

\section{Data collection}

The data was collected by the principal investigator from a computer database. They were then retranscribed on a standardized Microsoft Excel ${ }^{\circledR}$ file

\section{RESULTS}

\section{Population}

In total, 74 patients were included. 84 positive samples were identified, with the presence of 10 duplicates that were removed.

- Among these patients, the female sex was predominant with 48 women for 26 men

- The median age was 80 years [ 18-100].

- The distribution of patients between a service under a medical care and a surgical service is shown in Figure 5

- The median duration of hospitalization was 10 days, with a minimum duration of less than 24 hours during outpatient care, and a maximum duration of 100 days. The average duration of hospitalization was 18 days.

- The average time between admission to hospital and the occurrence of infection was 5.5 days. The median was 1 day [0-33].

It should be noted that the residence data of patients residing in the EHPAD who were not hospitalized at the HC of Quimper were not selected.

\section{ESBL risk factors}

Risk factors for EBLSE infection are shown in Table $\mathbf{1 .}$

Urinary colonization prior to EBLSE was known in 6 patients (5 patients had colonization with E. Coli, 1 with Citrobacter freundii); 2 patients had an E. coli digestive portage. 


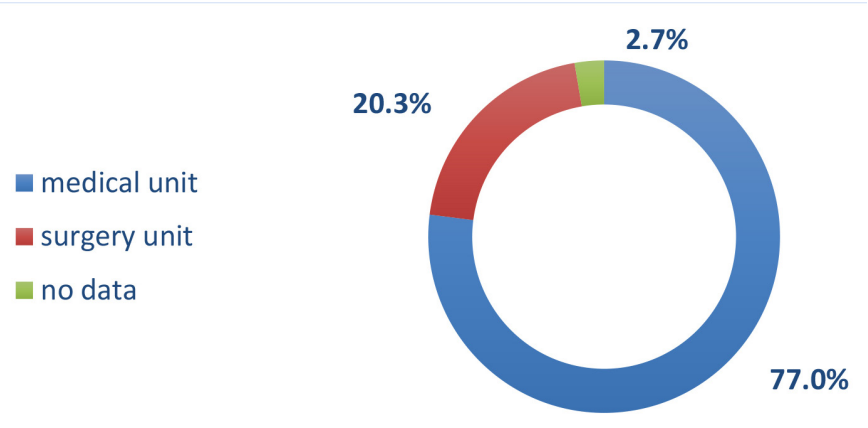

Figure 5. Patients sectorization

Table 1. Risk Factors for EBLSE Infection of Patients

\begin{tabular}{|l|c|}
\hline \multicolumn{1}{|c|}{ Risk factors } & patients : $\mathbf{n} ; \mathbf{~}$ \\
\hline Advanced age $\geq 80$ years & $38 ; 51.4 \%$ \\
\hline EHPAD (life in an institution) & $22 ; 29.7 \%$ \\
\hline History of infection and / or carriage of EBLSE & $16 ; 21.6 \%$ \\
\hline Long-term hospitalization $\geq 14$ days & $30 ; 40.5 \%$ \\
\hline Hospitalization in the year preceding admission & $38 ; 51.4 \%$ \\
\hline Travel abroad during the year & $4 ; 5.4 \% *$ \\
\hline Medical device in place & $7 ; 9.5 \%$ \\
\hline
\end{tabular}

* 30 missing data

Eight patients had a known history of infection with ESBL E (4 urinary infection and one bile liquid infection with $E$. coli, one urinary infection with Citrobacter freundii, one urinary tract infection with Enterobacter cloacae complex (E. cloacae complex), one peritonitis Citrobacter freundii).

Regarding the presence of foreign material, 2 patients had a Percutaneous Implantable Chamber (PIC), a patient with an orthopedic prosthesis, 2 patients with endovascular material such as aortic stents and a dialysis catheter, 2 patients with invasive urological material. cysto- catheter type and JJ probe. It should be noted that the presence of an indwelling urinary catheter has not been noted. In total, 7 patients $(9.5 \%)$ had a medical device in place.

3. The infectious episode

The different types of infections that have occurred are shown in Figure 6.

46 patients $(62.2 \%)$ had clinical signs of urinary tract infection. 9 patients (12.3\%) had an intra-abdominal infection, 7 patients (9.4\%) had signs of low respiratory infections

The different types of sampling that showed an EBLSE are shown in Figure 7.

The allocation of enterobacteriaceae species is shown in Table 2.

E. coli was the most common uropathogen. It was present in 54 cases (41 ECBU, 9 blood cultures, other samples not detailed). The second specie most frequently isolated was E. cloacae complex present in 12 cases (8 ECBU, 4 blood cultures, other samples not detailed). There were 4 cases of $K$. pneumoniae infection.
5

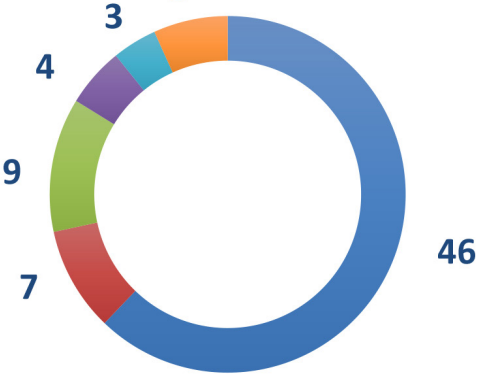

\begin{abstract}
—urinary tract infections aulmonary infections
- intra abdominal infections $\quad$ endovascular infections
\end{abstract}

asteoarticulatory infections u indeterminated

Figure 6. Nature of infectious episodes

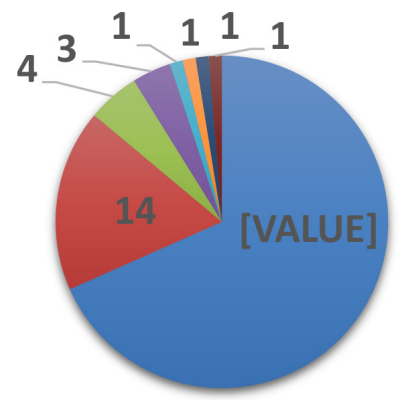

$$
\begin{aligned}
& \text { urine } \\
& \text { peritoneal fluid } \\
& \text { hepatic biopsy } \\
& \text { bone }
\end{aligned}
$$$$
\text { alood }
$$

- bronchopulmonary lavage$$
\text { stool }
$$

prosthetic material

Figure 7. Collection types

Table 2. Distribution of Enterobacteriaceae

\begin{tabular}{|l|c|c|c|}
\hline \multicolumn{1}{|c|}{ species } & n; \% & urine & $\begin{array}{c}\text { Blood } \\
\text { cultures }\end{array}$ \\
\hline E. coli & $54(72.9 \%)$ & 41 & 9 \\
\hline E. cloacae complex & $12(16.2 \%)$ & 8 & 4 \\
\hline K. pneumoniae & $4(5.4 \%)$ & 2 & 1 \\
\hline Citrobacter freundii & $2(2.7 \%)$ & 2 & - \\
\hline Citrobacter koseri & $1(1.4 \%)$ & 1 & - \\
\hline Salmonella enterica & $1(1.4 \%)$ & - & - \\
\hline
\end{tabular}

Bacteremia was present in 14 cases (19.0\%).

The susceptibility profiles of the different molecules tested are represented Figure 8. Only one strain had a carbapenem resistance profile (E. cloacae complex). The sensitivity profile of the 3 most common bacteria is described in Figure 9.

It is estimated that the infection was associated with care in at least 29 cases: 


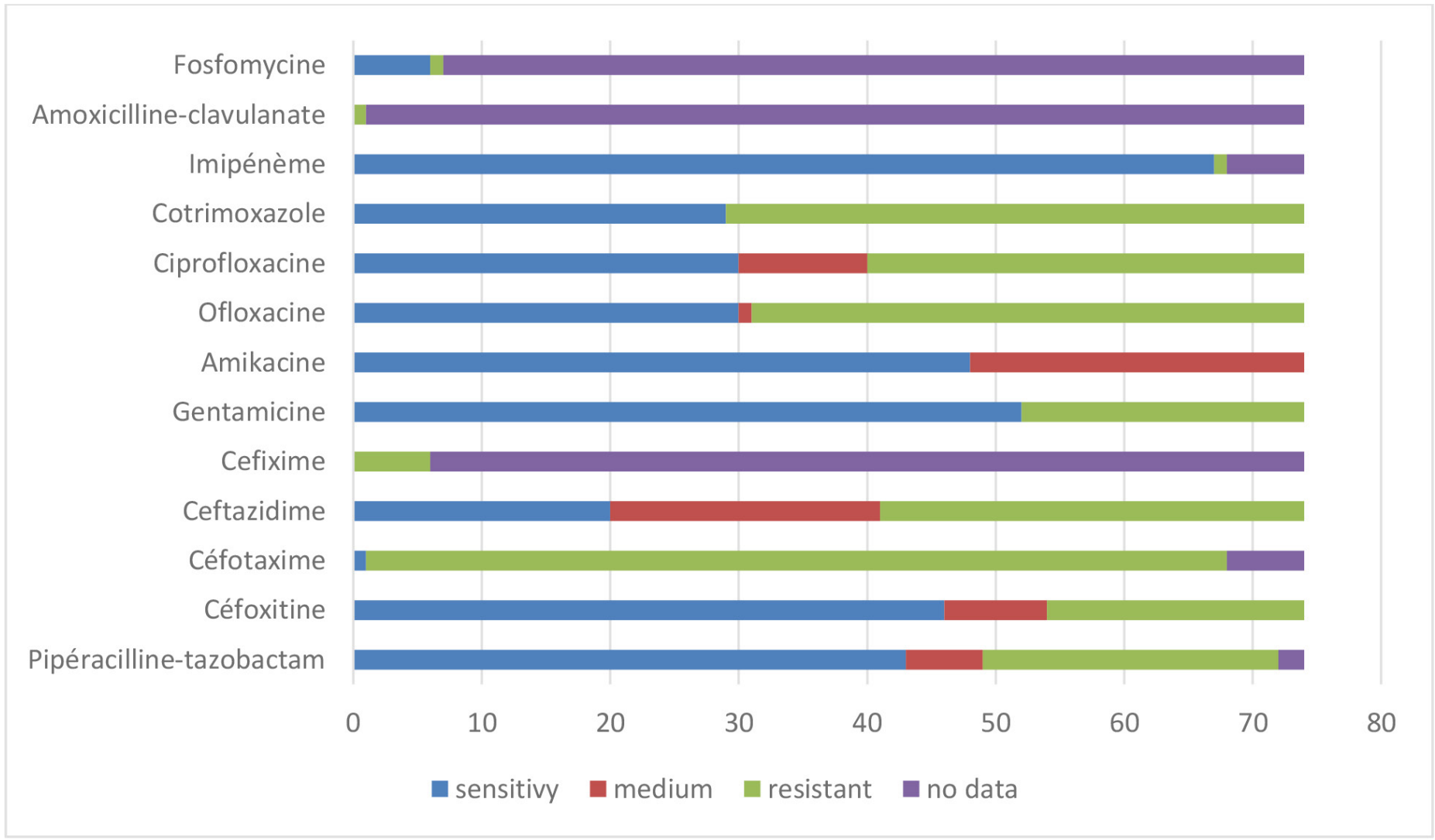

Figure 8. Sensitivity profile of antibiotic strains

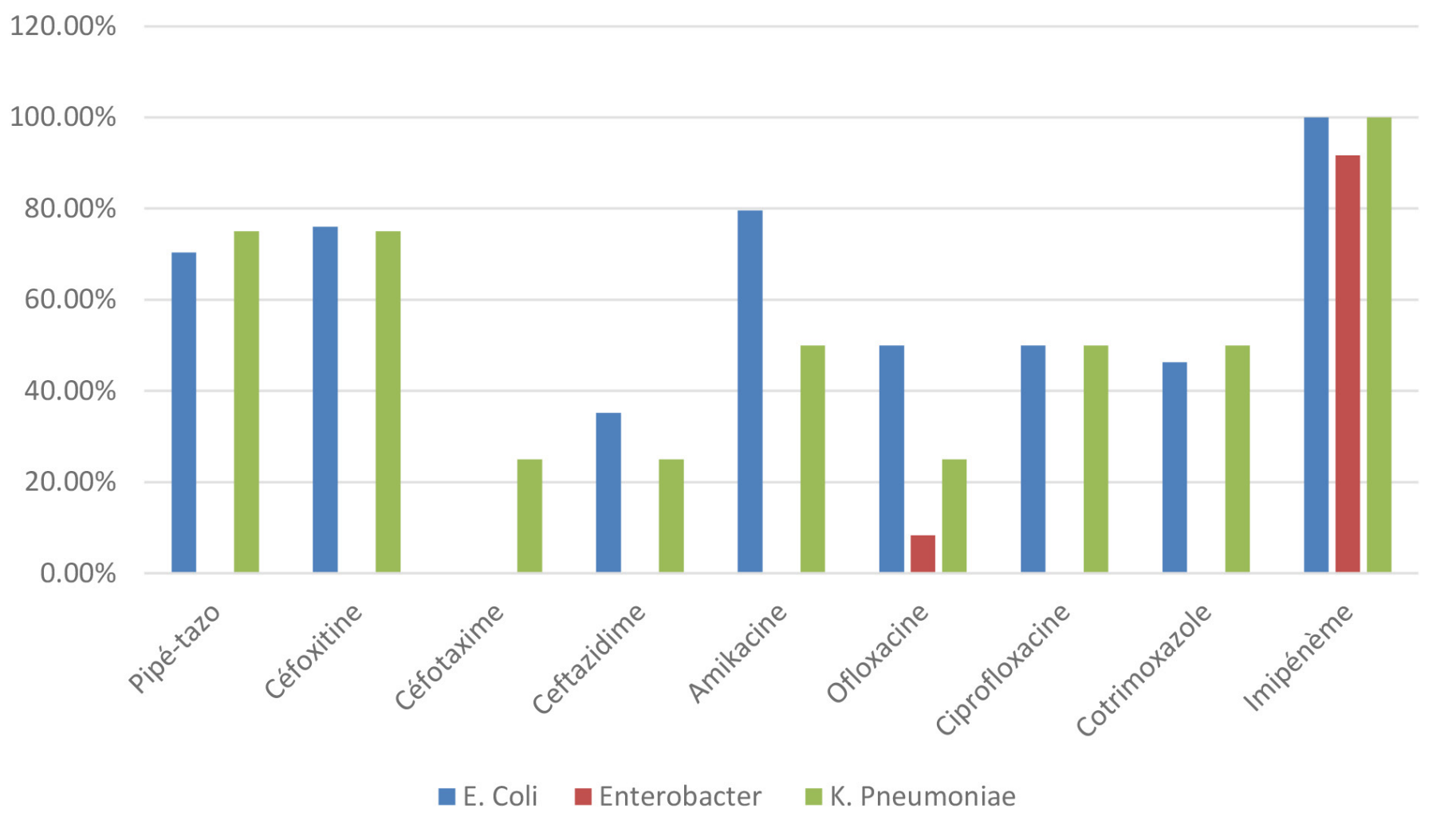

Figure 9. Sensitivity profile of the 3 most frequently isolated bacteria 
Patients infected with EBLSE $\mathrm{n}=74$

- $\quad$ ESBL possibly acquired (delay $>48$ hours) $\mathrm{n}=29$

- Carriage of Imported ESBL (time $\leq 48$ hours) $\mathrm{n}=36$

Missing data $\mathrm{n}=9$

\section{Treatment}

A total of 47 patients (63.5\%) received antibiotic therapy. 15 patients did not receive anti-infectious treatment. Data was missing for 12 patients.

- Probabilistic antibiotic therapy

The strategies for choosing initial antibiotic therapy are detailed in Table 3.

Table 3 . Probabilistic antibiotic therapy.

\begin{tabular}{|l|c|}
\hline \multicolumn{1}{|c|}{ antibiotics } & All infections, $\mathbf{n} \%$ \\
\hline amoxicillin & $2 ; 3.2 \%$ \\
\hline Amoxicillin-clavulanate & $3 ; 4.8 \%$ \\
\hline ceftriaxone & $12 ; 19.5 \%$ \\
\hline Ceftriaxone + metronidazole & $5 ; 8.1 \%$ \\
\hline Piperacillin-tazobactam & $3 ; 4.8 \%$ \\
\hline Piperacillin Tazo + aminoglycoside & $1 ; 1.6 \%$ \\
\hline Piperacillin Tazo + glycopeptide & $1 ; 1.6 \%$ \\
\hline Aztreonam-linezolid & $1 ; 1.6 \%$ \\
\hline cefepime & $1 ; 1.6 \%$ \\
\hline carbapenems & $7 ; 11.3 \%$ \\
\hline Carbapenem + aminoglycoside & $2 ; 3.2 \%$ \\
\hline fluoroquinolones & $3 ; 4.8 \%$ \\
\hline pristinamycin & $1 ; 1.6 \%$ \\
\hline Fosfomycine trometamol & $2 ; 3.2 \%$ \\
\hline cotrimoxazole & $2 ; 3.2 \%$ \\
\hline furans & $15 \% 24.3 \%$ \\
\hline No treatment & \\
\hline Total & \\
\hline
\end{tabular}

The most used molecule was ceftriaxone. Of the patients who received the piperacillin-tazobactam combination, none had a known history of EBLSE carriage but their period of hospitalization was $>48$ hours. Among patients who received imipenem, one patient had a history of urinary infection with E. coli ESBL and another with Citrobacter freundii ESBL, a patient had a history of peritonis with Citrobacter freundii; the other patients had been hospitalized for more than 48 hours.

Probabilistic antibiotic therapy was considered active in 21 cases out of 47 treated patients, ie $44.7 \%$ of cases.

- Documented antibiotic therapy
After reassessment, antibiotic therapy was modified in 19 cases. The choice of antibiotherapy after microbiological documentation and knowledge of the antibiogram are detailed Table 4.

Table 4. Antibiotic for second line

\begin{tabular}{|l|c|}
\hline \multicolumn{1}{|c|}{ antibiotics } & $\mathbf{n}=\mathbf{1 9} ; \%$ \\
\hline Amoxicillin- clavulanate & $4 ; 21.1 \%$ \\
\hline Ceftazidime-ofloxacin & $1 ; 5.3 \%$ \\
\hline Piperacillin Tazo + aminoglycoside & $1 ; 5.3 \%$ \\
\hline Fluoroquinolone alone & $5 ; 26.2 \%$ \\
\hline cotrimoxazole & $1 ; 5.3 \%$ \\
\hline carbapenems & $5 ; 26.2 \%$ \\
\hline furans & $1 ; 5.3 \%$ \\
\hline temocillin & $1 ; 5.3 \%$ \\
\hline
\end{tabular}

Of the 19 patients who received a therapeutic modification, only one died.

A third generation cephalosporin $(\mathrm{C} 3 \mathrm{G})$, ceftriaxone, was used in probabilistic for 17 patients. Among them, a therapeutic modification took place for 12 patients. It should be noted that of the 17 patients treated with C3G, 2 died (one left on unsuitable probabilistic antibiotic therapy), 2 had deep abscesses, one patient had severe sepsis. Inadequate initial treatment seemed effective for 4 patients.

- Provision of the opinion of an infectious disease specialist

In 28 cases only, treatments were advised by an infectious diseases specialist, leading to the prescription of a carbapenem in 9 cases, a $\beta$ - lactamin - $\beta$ - lactamases inhibitors (BL-IBL) in 8 cases ( 3 times amoxicillin-clavulanic acid and 5 times piperacillin-tazobactam), one fluoroquinolone in 3 cases, cotrimoxazole in 1 case.

De-escalation strategies were more frequent when infectious advice was given.

It should be noted that 5 of 28 patients carried a medical device. Of these 28 patients, 3 died as a result of EBLSE infection.

- Processing times

It was difficult to evaluate treatment times because of their heterogeneity due to the various pathologies and the many missing data because they were not found in patients' files. However, the median duration of treatment at 10 days can be evaluated with a minimum duration of one day and a maximum duration of approximately 90 days (especially in the osteoarticulatory infections), for an average duration of 12.9 days, all infections combined.

\section{Evolution}

The evolution was favorable for 64 patients (2 patients lost to follow-up). Among the complications found : 8 deaths, 2 septic shock favorable, unspecified epilepsy with imipenem, two patients presented deep abscesses, a patient presented with clostridium difficile colitis. 
4 patients died while initial antibiotic therapy was considered active.

\section{Discussion}

In general, the choice of antimicrobial therapy taking into account microbiological data is essential : mortality is higher if effective treatment initiation is delayed or inactive on EBLSE. Carbapenem treatments are associated with the lowest mortality. But the use of this class of antibiotics in the presence of ESBL also promotes the emergence of other enzymes, the metallo- beta-lactamases that hydrolyze carbapenems (CPE) and render them ineffective. In order to limit this risk, the use of antibiotic molecules that are alternative to carbapenems and frequently active in vitro on EBLSE has been encouraged. Some $\beta$ - lactams (such as cephamycins, temocillin, aztreonam (AZT), the mecillinam, the BL-IBL associations or C3G) retain in vitro activity on certain ESBLE [13]. Since 2009, CA-SFM has modified the critical levels of C3G and AZT for Enterobacteriaceae based on proposals made by the European Committee on Antimicrobial susceptibility Testing (EUCAST) [14]. Thus, a strain is, since 2009, categorized Sensitive (S) when the MIC of C3G and AZT is $\leq 1 \mathrm{mg}$ / L whereas it was previously when CMI was $\leq 4 \mathrm{mg} / \mathrm{L}$. To encourage these carbapenem saving practices, the CASFM issued a press release in 2011 recommending no longer interpretative reading for the codification of Enterobacteriaceae strains having acquired mechanisms of resistance to $\mathrm{C} 3 \mathrm{G}$ and AZT, but while continuing to detect the ESBL to monitor their evolution. But the clinical efficacy of these molecules compared to carbapenems is debated [15].

The main objective of this study was to evaluate in current practice the therapies administered and their efficacy in the case of EBLSE documented infection, and in particular to study alternative molecule to carbapenem. The secondary objectives were to identify the risk factors presented by these patients and to study the management of the infectious episode.

This work is original insofar as it is concerned with the current practice of a resistant germ infection in a secondary HC. It is heterogeneous because it covers several sites of infection and several bacterial species.

In this study, the risk factors for EBLSE infection are generally those described in the literature, but with several limitations.

Patient comorbidities and history of immunosuppression have not been identified, nor the concept of recurrent urinary tract infections. Regarding the medical device in place, the presence of a current bladder catheter or in the previous 3 months was not sought. Hospitalization in the year preceding admission was sought but was not mentioned prior treatment with the $\beta$-ctamines and / or fluoroquinolones. [16] There were few patients who traveled to areas known to be at risk of colonization and / or EBLSE infection. In addition, the search for an earlier colonization resistant germ was made only on the Quimper hospital and therefore remains very limited. The children have not been included. More than half of the patients had an advanced age $\geq 80$ years and almost a third lived in EHPAD. The predominance of women and urinary tract infections at EBLSE are consistent with the literature. Regarding the community or nosocomial origin, the data are also in agreement with the literature, confirming that the EBLSE are far from being present only in the hospital [17].

During the year 2016, 108 adult patients had a positive diagnostic specimen at EBLSE. Only 74 patients were included in this study. In 2015 , we find a number of patients equivalent with a positive diagnostic sample at EBLSE. Between 2015 and 2016, hospital of Quimper has seen its consumption of antibiotics increase, especially that of C3G and monobactams (cf. report ConsoRes 2016, evaluating antibiotic consumption and resistance monitoring s in Quimper HC, compared to institutions same size, Annex 5). Amoxicillin- clavulanate remains the most widely used antibiotic. On the other hand, there is less consumption of fluoroquinolones and carbapenems.

If we look at the 3 main enterobacteria found in this study, we note that the percentage of resistance of E. coli and K. pneumoniae to cefotaxime is decreasing between 2015 and 2016. On the other hand, it is increasing for Enterobacter cloacae. Resistance to fluoroquinolones also appears to be increasing for E. coli and E. cloacae. Carbapenem resistance is dissociated with a decrease in overall resistance to imipenem but an increase in resistance to ertapenem.

The question of antibiotic therapy of choice in EBLSE infections remains debated. In this work, the most used probabilistic antibiotic therapy was a C3G, often prescribed as soon as the patient was admitted to the hospital and most of the time via the Emergency Reception Service, followed by a carbapenem. AZT has hardly been used. C3G such as ceftriaxone and cefotaxime remain molecules often used in probabilistic. Here, ceftriaxone appeared to be effective in only 4 cases. Each time, it was an urinary tract infection with E. coli, no infectious advice was taken, and the antibiogram showed " not rendered " in 2 cases and " $\mathrm{R}$ " in the other 2 cases. AZT was used once, in the case of an osteoarticulatory infection with E. cloacae complex with favorable evolution. After microbiological documentation, only one patient received C3G (ceftazidime), in combination with a fluoroquinolone, as part of an E. coli PIC infection and after consultation with an infectious disease specialist. Successful treatment of EBLSE with C3G has been reported in clinical cases or retrospective studies [18, 19]. It is nevertheless a small number of patients and mixed success. Their use seems possible in the case of mild and low inoculum EBLSE infections due to strains with low minimum inhibitory concentration (MIC). Some literature data suggest that when probabilistic C3G therapy is compared to probabilistic carbapenem or BL-IBL treatment, the mortality rate is higher with C3G therapy [20].

In this study, BL-IBLs such as amoxicillin- clavulanate and piperacillin-tazobactam were frequently used and mostly effective in urinary tract infections, in two cases of low respiratory tract infections, one with E. Coli and the other with K. pneumoniae, and in one case of intra-abdominal E. coli infection, whether in probabilistic or microbiological documentation. In re-evaluating the crude results of antibiograms for BL-IBL, EUCAST has also facilitated their potential therapeutic use for susceptible categorized strains, but only in cases of urinary tract infection and / or bacteremia with a urinary origin [14].

The carbapenems were prescribed as empiric antibiotic therapy in patients when ESBL colonization was known, in appropriate antibiotic 
therapy in case of failure or resistance to initial treatment. These attitudes are consistent with the recommendations for the proper use of carbapenems. In the litterature, some studies found a carbapenem superiority compared to BL-IBL [21] while others conclude noninferiority of BL-IBL on carbapenems $[22,23]$. These results are encouraging to limit the emergence of carbapenemases. Nevertheless, one should be wary of the inoculum effect observed with piperacillintazobactam and which could lead to clinical failures whereas the MIC in vitro was low with a labeled antibiotic [24]. Current evidence suggests that the use of BL-IBL for the treatment of EBLSE infections would be effective, if certain conditions are met such as a low MIC, a low inoculum and a sufficient antibiotic dose with extended infusion.

No molecule of the cephamycin class was used. Cefoxitin has in vitro efficacy against ESBL but its use still seems limited because its clinical and microbiological efficacy has not been evaluated. A study is in progress (COLIFOX Trial) evaluating the non-inferiority of cefoxitin versus imipenem in the treatment of urinary tract infections and ESBL-sensitive E. coli bacteremia in vitro.

Other antibiotics do not belong to the family of the $\beta$ - lactamine were also used : cotrimoxazole, fluoroquinolones, furans and fosfomycin. These last two molecules can be the treatment of choice for urinary infections such as cystitis [25]. However, antibiograms were poorly reported for fosfomycin and furans in our study. Cotrimoxazole has low sensitivity rate for Enterobacteriaceae in general and especially for ESBLE. Its sensitivity rate here is less than $30 \%$ for all strains. However, they may be of great interest in the future, given the emergence of resistance. Fluoroquinolones have rarely been used as first, but turn out as frequent as carbapenems in second line. However, the use of carbapenems is sometimes necessary and the only recourse, when one focuses on resistance profiles to the class of fluoroquinolones.

The opinion of an infectious disease specialist is very often necessary because of the complexity of the clinical and microbiological care. Strategies for climbing or de-escalating must be argued all the more in the presence of resistant germs. In order to limit the emergence of resistance, it seems essential to justify broad-spectrum antibiotic prescriptions in complex and serious cases as well as in simple cases.

The analysis of the treatment durations finds very heterogeneous results in connection with various pathologies ranging from urinary infection to osteoarticular infection. Whenever a carbapenem was prescribed, an infectious advice had been taken beforehand, limiting the inappropriate duration of treatment.

Eight patients died during the course of EBLSE infection, ie 10.8\%. This figure appears to be lower than the death rate described in the literature (between $20 \%$ and $60 \%$ ) [26,27], probably due to the fact that this cohort is only part of the population that has had EBLSE infection during the year 2016 in a hospital center. In addition, the literature often reports serious infections; here, community infections have also been reported.

Although this study is limited by the fact of its retrospective, monocentric character, with sometimes inaccurate data and without a control group, it does however highlight quite different therapeutic possibilities in case of EBLSE infection. The carbapenems are far from the only effective molecules and their need is not evident in the literature. Prospective studies are expected to try to close the debate. Progress can still be made, particularly on the management of infectious episodes, by improving the communication between emergency-specialists-infectiologists-organ specialists and microbiologists, by being vigilant with fragile patients who have undergone several prior antibiotics and potentially carriers of germs (s) resistant, so that hygiene measures are put in place upon admission to hospital, which is rarely the case.

\section{Conclusion}

ESBL enterobacterial infections represent a major therapeutic challenge. Some molecules seem to position themselves as therapeutic alternatives to carbapenems, including BL-IBL, or even other classes after microbiological documentation, if we follow the recommendations of EUCAST and CA-SFM.

In view of the evolution of bacterial resistance and the lack of innovation in antibiotic therapy, the safeguarding of antibiotics requires a reasoned use of the molecules conventionally used, a new exploitation of previously neglected molecules, an innovation in terms of association and improvement in preventing the transmission of multidrug-resistant bacteria (28).

\section{ABREVIATIONS LIST}

AZT : aztreonam

BL-IBL: $\beta$ - lactam - $\beta$ - lactamases inhibitors

ESBL : extended spectrum $\beta$ - lactamase

BMR: multi-resistant bacterium

C3G : C éphalosporines ${ }^{3 r d}$ generation

CA-SFM : Committee of the antibiogram of the French Society of Microbiology

CCLIN: Coordination Center for the Fight against Nosocomial Infections

CIP : Percutaneous Implantable Chamber

CPE : carbapenemase-producing Enterobacteriaceae

CTX-M: cefotaxime

EBLSE: Enterobacteria producing ESBL

ECBU: Cyto-bacteriological examination of urine

E. cloacae : Enterobacter cloacae

E. Coli : Escherichia coli

EHPAD: Accommodation facility for dependent elderly people

EPC : Enterobacteria producing carbapenemases

EUCAST : European Committee on Antimicrobial Susceptibility Testing

HC : hospital center

"I “ : Intermediate

JH : Day of Hospitalization

K. pneumoniae : Klebsiella pneumoniae

MIC : minimum inhibitory concentration 
MRSA : Staphylococcus Aureus Meticillin Resistant

RAISIN: Network of Alert, Investigation and Surveillance of Nosocomial Infections

"R" : Resistant

"S" : Sensible

MRSA: Staphylococcus Aureus Meticillin Resistant

\section{References}

1. Pitout JD, Laupland KB (2008) Extended-spectrum betalactamase -producing Enterobacteriaceae : An emerging public-health concern. Lancet Infect Dis 8: 159-66.

2. Arias CA, Murray BE (2009) Antibiotic-resistant bugs in the 21st century- A clinical super-challenge. $N$ Engl J Med 360: 439-43.

3. European Center for Disease Prevention and Control. Carbapenemase -producing bacteria in Europe: Interim results from the European Survey on carbapenemase producing Enterobacteriaceae (EuSCAPE) project. Stockholm: ECDC; 2013

4. S. Vora, R. Auckenthaler (2009) What does mean " broad spectrum beta- lactamases " in practice? Rev Med Switzerland 5 : 1991-4.

5. Knothe H, Shah P, Krcmery V, Antal M, Mitsuhashi S (1983) Transferable resistance to cefotaxime, cefoxitin, cefamandole and cefuroxime in clinical isolates of Klebsiella pneumoniae and Serratia marcescens . Infection 11: 315-317.

6. Sirot D, Sirot J, Labia R, Morand A, Courvalin P, Darfeuille- Michaud A, et al. (1987) Transferable resistance to third-generation cephalosporins in clinical isolates of Klebsiella pneumoniae: identification of CTX-1, a novel beta-lactamase. $J$ Antimicrob Chemother 20: 323-334

7. Hull TM, Baquero F, Canton R (2008) Increasing prevalence of ESBL-producing Enterobacteriaceae in Europe. Euro Surveill Bull Eur On Mal Transm Eur Commun Dis Bull 2008 : 13.

8. Zahar JR, Ball E, Schnell D, Lantern F, Mechai F, Mass V, et al. (2009) [Extension of beta-lactamases producing bacteria is a worldwide concern]. MS Sci Medicine 25: 939-944.

9. Network of alert, investigation and surveillance of nosocomial infections, RAISIN 2015.

10. Paterson DL, Bonomo RA (2005) Extended-spectrum beta-lactamases: a clinical update. Clin Microbiol Rev 18: 657-686.

11. Proportion of invasive E. coli strains resistant to 3rd generation cephalosporins, Europe, 2015 . European Antimicrobial Resistance Surveillance Network (EARSNet) .

12. Definition of Care Associated Infections - Technical Committee for Nosocomial Infections and Care-Related Infections. Ministry of Health, Youth and Sports (2007).

13. Fournier D, Chirouze C, Leroy J, Cholley P, Talon D, Plesiat P, et al. (2013) Alternatives to carbapenems in ESBL- producing Escherichia coli infections. Medicine and infectious diseases 43: 62-6.

14. Leclercq R, Canton R, Brown DF, Giske CG, Heisig P, MacGowan AP, et al. (2013) EUCAST expert rules in antimicrobial susceptibility testing. Clinical Microbiology and Infection: The official publication of the European Society of Clinical Microbiology and Infectious Diseases 19: 141-60.

15. Harris PN, Tambyah PA, Paterson DL (2015) beta-lactam and beta-lactamase inhibitor compounds in the treatment of extended-spectrum beta-lactamase producing Enterobacteriaceae : time for a reappraisal in the era of few antibiotic options? Tea Lancet Infectious diseases 15: 475-85.

16. Rodríguez-Baño J, Alcalá JC, Cisneros JM, Grill F, Oliver A, Horcajada JP, et al. (2008) Community infections caused by extended-spectrum beta-lactamaseproducing Escherichia coli. Arch Intern Med 168: 1897-1902 .

17. Saltoglu N, Karali R, Yemisen M, Ozaras R, Balkan, II, Mete B, et al. (2015) [Escherichia coli and antimicrobial activities]. International journal of clinical practice. 2015.

18. Paterson DL, Ko WC, Von Gottberg A, Casellas JM, Mulazimoglu L, Klugman $\mathrm{KP}$, et al. (2001) Outcome of cephalosporin treatment for serious infections due to apparently susceptible producing extended-spectrum beta-lactamases: implications for 69 the clinical microbiology laboratory. Journal of clinical microbiology 39: 2206-12.

19. Rodriguez- Bano J, Picon E, Navarro MD, Lopez-Cerero L, Pascual A (2012) Impact of changes in CLSI and EUCAST breakpoints for susceptibility in bloodstream infections due to extended-spectrum beta-lactamase-producing Escherichia coli. Clinical Microbiology and Infection: The official publication of the European Society of Clinical Microbiology and Infectious Diseases. 18: 894-900.
20. Rodriguez- Bano J, Picon E, Gijon P, Hernandez J, Ruiz M, Pena C, et al. (2010) Community onset bacteremia due to extended-spectrum beta- lactamase-producing Escherichia coli: risk factors and prognosis. Clinical Infectious Diseases: an official publication of the Infectious Diseases Society of America. 2010; 50: 40-8.

21. Ofer- Friedman H, Shefler C, Sharma S, Tirosh A, Tal-Jasper R, Kandipalli D, et al (2015) Carbapenems Versus Piperacillin- Tazobactam for Bloodstream Infections of Nonurinary Source Caused by Extended-Spectrum Beta-Lactamase-Producing Enterobacteriaceae. Infect Control Hosp Epidemiol 2015: 1-5.

22. Harris PN, Yin M, Jureen R, Chew J, Ali J, Paynter S, et al. (2015) Comparable outcomes for beta-lactam / beta-lactamase inhibitor combinations and carbapenems in definitive treatment of bloodstream infections caused by cefotaxime-resistant Escherichia coli or Klebsiella pneumoniae. Antimicrobial resistance and infection control 4: 14

23. Vardakas KZ, Tansarli GS, Rafailidis PI, Falagas ME (2012) Carbapenems versus alternative antibiotics for the treatment of bacteraemia due to Enterobacteriaceae producing extended-spectrum beta-lactamases: a systematic review and metaanalysis. J Antimicrob Chemother 67: 2793-803.

24. Thomson K, Moland E (2001) Cefepime, piperacillin- tazobactam, and the inoculum effect in tests with extended-spectrum beta-lactamase-producing Enterobacteriaceae. Antimicrobial agents and chemotherapy 45: 3548-54.

25. Sultan A, Rizvi M, F Khan, Sami H, Shukla I, Khan HM (2015) Increasing antimicrobial resistance among uropathogens : Is fosfomycin the answer? Urology annals 7 (1): 26-30.

26. Lew KY, Ng TM, Tan M, SH Tan, Lew EL, Ling LM, et al. (2015) Safety and clinical outcomes of carbapenem de-escalation as part of an antimicrobial stewardship program in an ESBL-endemic setting. J Antimicrob Chemother 70: 1219-1225.

27. Peralta G, Lamelo M, Alvarez-Garcia P, Velasco M, Delgado A, Horcajada J, et al. (2012) Impact of empirical treatment in extended-spectrum beta-lactamaseproducing Escherichia coli and Klebsiella spp. bacteremia. A multicentric cohort study . BMC infectious diseases 12: 245.

28. European Food Safety Authority and European Center for Disease Prevention and Control (2019) The European Union summary report on microbial resistance in zoonotic and indicator bacteria for humans, animals and food in 2017. EFSA Journal 17: 5598

\section{Citation:}

Margaux Geier (2019) Enterobacteria Producing Extended Spectrum BetaLactamases : 74 Cases in a General Hospital in France. Internal Med Res Open $J$ Volume 4(1): 1-13. 


\section{Annexure}

\section{Annex 1: Phenotypes of resistance of enterobacteria to $\beta$ - lactams}

- Natural resistances of enterobacteria to $\beta$ - lactams:

Enterobacteria are naturally resistant to penicillins $\mathrm{G}$ and $\mathrm{M}$, depending on additional resistance to other $\beta$ lactam antibiotics, they are classified into four groups:

\begin{tabular}{|c|c|c|c|c|}
\hline Group of $\beta$ - lactamines & Group 1 & Group 2 & Group 3 & Group 4 \\
\hline $\begin{array}{l}\text { Main types of enterobacteria } \\
\text { encountered in hospitals }\end{array}$ & $\begin{array}{l}\text { E. coli } \\
\text { Proteus mirabilis } \\
\text { Salmonella Shigella }\end{array}$ & $\begin{array}{l}\text { Klebsiella Citrobacter } \\
\text { koseri }\end{array}$ & $\begin{array}{l}\text { Enterobacter } \\
\text { Serratia } \\
\text { Morganella } \\
\text { Providencia Citrobacter } \\
\text { freundii }\end{array}$ & Yersinia \\
\hline aminopenicillins & S & $\mathbf{R}$ & $\mathbf{R}$ & $\mathbf{R}$ \\
\hline Carboxypénicillines & S & $\mathbf{R}$ & S & $\mathbf{R}$ \\
\hline ureidopenicillins & S & $I / R$ & S & $\mathbf{I} / \mathbf{R}$ \\
\hline $\mathrm{C} 1 \mathrm{G}$ & S & S & $\mathbf{R}$ & $\mathbf{R}$ \\
\hline C3G & S & S & S & S \\
\hline carbapenems & S & S & S & S \\
\hline Mechanisms of resistance & Absence of $\beta$ - lactamase & Penicillinase at low level & Cephalosporinase at low level & Penicillinase + cephalosporinase \\
\hline
\end{tabular}

- Resistances acquired from $\beta$ - $\underline{\text { lactam }} \underline{\text { enterobacteria : }}$

Presentation of resistance phenotypes of enterobacteria to $\beta$ - lactams :

\begin{tabular}{|c|c|c|c|c|c|c|}
\hline Antibiotic markers & $\begin{array}{c}\text { Low level } \\
\text { penicillinase }\end{array}$ & $\begin{array}{c}\text { Penicillinase } \\
\text { high level }\end{array}$ & $\begin{array}{c}\text { Penicillinase } \\
\text { resistant to } \\
\text { I } \beta \text { L }\end{array}$ & $\begin{array}{c}\text { Cephalosporinase low } \\
\text { level }\end{array}$ & $\begin{array}{l}\text { Cephalosporinase high } \\
\text { level }\end{array}$ & ESBL $^{1}$ \\
\hline $\begin{array}{l}\text { amoxicillin } \\
\text { AMX aminopenicillin }\end{array}$ & $\mathrm{R}$ & $\mathrm{R}$ & $\mathrm{R}$ & $\mathrm{R}$ & $\mathrm{R}$ & $\mathrm{R}$ \\
\hline $\begin{array}{l}\text { amoxicillin }+ \\
\text { Ac.clavulanique } \\
\text { AMC aminopenicillin }+\mathrm{I} \beta \mathrm{L}\end{array}$ & S & $I / R$ & $\mathrm{R}$ & $\mathrm{R}^{2}$ & $\mathrm{R}^{2}$ & $\mathrm{R}^{3}$ \\
\hline $\begin{array}{l}\text { ticarcillin } \\
\text { TIC carboxypenicillin }\end{array}$ & $\mathrm{R}$ & $\mathrm{R}$ & $\mathrm{R}$ & S & $\mathrm{R}$ & $\mathrm{R}$ \\
\hline $\begin{array}{l}\text { mecillinam } \\
\text { MEC aminidopenicillin }\end{array}$ & S & $\mathrm{R}$ & $\mathrm{R}$ & S & S & $\mathrm{R}$ \\
\hline $\begin{array}{l}\text { cephalothin } \\
\mathrm{CF}(\mathrm{C} 1 \mathrm{G})\end{array}$ & S & $\mathrm{R}$ & $\mathrm{S}$ & $\mathrm{R}$ & $\mathrm{R}$ & $\mathrm{R}$ \\
\hline $\begin{array}{l}\text { ceftazidime } \\
\text { CTX (C3G) }\end{array}$ & S & S & S & S & $\mathrm{R}$ & $\begin{array}{l}\mathrm{R} \text { or synergist } \\
-\mathrm{gy}^{4}\end{array}$ \\
\hline
\end{tabular}

1 : ESBL: $\beta$ - lactamase with extended spectrum. $\mid 2$ : I $\beta$ L: $\beta$ - lactamase inhibitors do not inhibit cephalosporinase s (cephalosporinases are nevertheless $\beta$ - lactamases) $\mid \mathbf{3}$ : Resistant strain sometimes intermediate, in all cases the inhibition diameter for the AMC is greater than that of the AMX. $\mid 4$ : some ESBLs can give an intermediate or sensitive profile with a C3G. The demonstration of synergy between clavulanic acid (AMC disk) and C3G not allow to conclude that the presence of an ESBL. 
Annex 2: Classification of $s \beta$ - lactamases according to amble:

- Serines transferases :

- Class A : (TEM, SHV, MEN, PES, PER, VEB, CTX-M ...)

Penicillinases, ESBL ; p lasmidic or chromosomal

- Class C : (CMY, DHA, FOX, MOX ...)

cephalosporinases; $\mathrm{c}$ hromosomiques or plasmid

- Class D : (OXA, PSE)

Oxacillinases p lasmidiques

- $\quad$ Metallo-enzymes: Class B (IMP, VIM, NDM-1)

carbapenemases

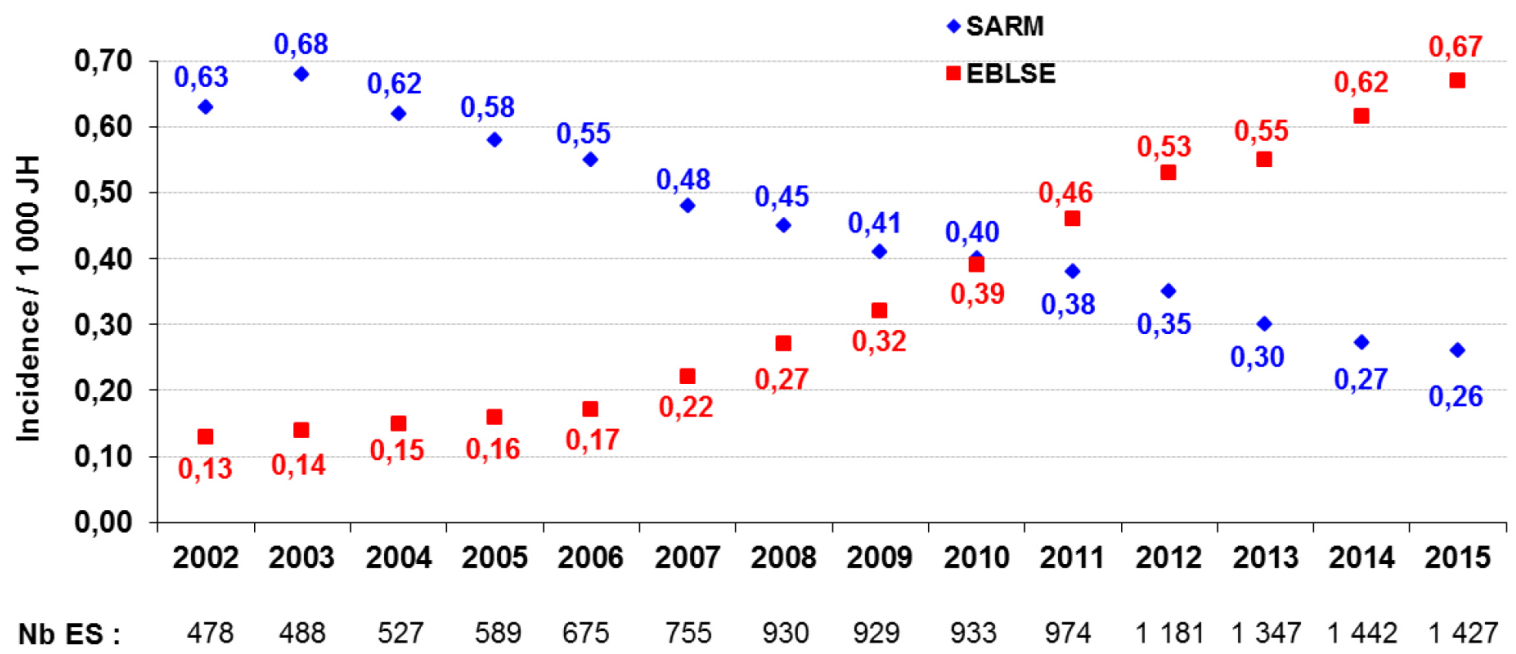

Annex 3: BMR-Raisin 2015- MRSA and EBLSE Incidence Densities per 1, 000 JH (overall incidence density per year) between 2002 and 2015 in France

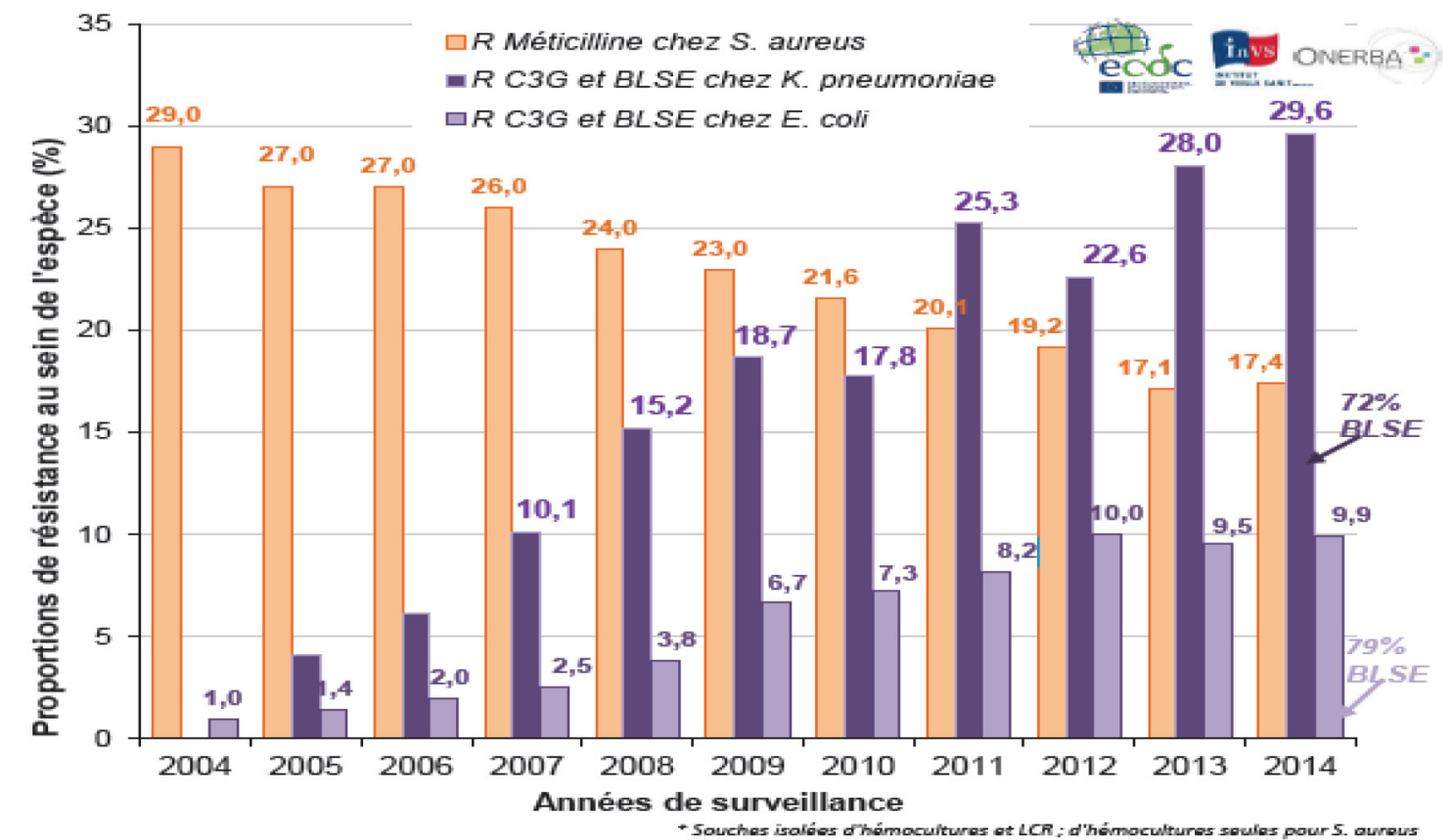

Annex 4 : Evolution of the methicillin resistance in S. aureus, and $3^{\text {rd }}$ generation cephalosporins in K. pneumoniae and E. Coli, France, 2004-2014, EARSNet France-InVS data 


\begin{tabular}{|l|r|r|r|}
\hline Famille & $\begin{array}{r}\text { Année 2015 } \\
\text { (DDJ/1000 } \mathbf{H}\end{array}$ & $\begin{array}{r}\text { Année 2016 } \\
\text { (DDJ/1000 } \mathbf{H}\end{array}$ & $\begin{array}{c}\text { Evolution } \\
\text { (\%) }\end{array}$ \\
\hline Pénicillines & 217,41 & 250,95 & $15,43 \%$ \\
\hline C 1G & 9,33 & 16,17 & $73,31 \%$ \\
\hline C 2G & 2,41 & 1,10 & $-54,36 \%$ \\
\hline C 3G & 37,34 & 41,91 & $1224 \%$ \\
\hline Autre cephalosporine & 0,53 & 0,17 & $-67,92 \%$ \\
\hline Carbapénèmes & 6,06 & 5,58 & $-7,92 \%$ \\
\hline Glycopeptides & 6,36 & 5,06 & $-20,44 \%$ \\
\hline Aminosides & 8,08 & 8,09 & $0,12 \%$ \\
\hline Quinolones & 37,96 & 36,48 & $-3,90 \%$ \\
\hline Macrolides et Lincosam \\
\hline Imidazolés & 13,77 & 13,66 & $-0,80 \% \mathbf{y}$ \\
\hline Monobactames & 13,24 & 13,23 & $-0,08 \% \mathbf{y}$ \\
\hline Sulfamides & 0,10 & 0,27 & $170,00 \%$ \\
\hline Autres & 10,20 & 7,38 & $-27,65 \%$ \\
\hline Anti-tuberculeux & 18,55 & 22,43 & $20,92 \%$ \\
\hline Tétracyclines & 4,40 & 3,42 & $-22,27 \%$ \\
\hline
\end{tabular}

Annex 5: ConsoRes report 2016, Quimper HQ 